\title{
Ocular hemorrhage due to warfarin: Five case reports
}

\author{
Sedat Koçak ${ }^{\mathrm{a}}$, Esma Erdemir ${ }^{\mathrm{b}}$, Birsen Ertekin ${ }^{\mathrm{c}^{*}}$, Başar Cander $^{\mathrm{a}}$, Cesareddin Dikmetaş ${ }^{\mathrm{a}}$ \\ ${ }^{a}$ Department of Emergency Medicine, Faculty of Medicine, Necmettin Erbakan University, Konya, Turkey \\ ${ }^{b}$ Department of Emergency Medicine, Kahramanmaras State Hospital, Kahramanmaras, Turkey \\ ${ }^{c}$ Department of Emergency Medicine, Beyhekim State Hospital, Konya, Turkey
}

ARTICLE INFO ABSTRACT

\section{Article History \\ Received $\quad 10 / 09 / 2013$ \\ Accepted $\quad 25 / 10 / 2013$}

\section{* Correspondence to:}

Birsen Ertekin

Department of Emergency,

Beyhekim State Hospital,

Konya, Turkey

e-mail: biceacil@hotmail.com

\begin{abstract}
An increase in the number of patients treated with anticoagulants has given rise to complications. In present study, five cases with ocular hemorrhages treated with warfarin were presented. Three men and two women applying to emergency between September 2008 and January 2012 were presented. Of cases, one was 55, and others were 65 and over. International normalized ratio (INR) values were, in turn, as follows: 5.40 in case $1,8.33$ in case 2, no coagulation in cases 3 and 4, and 5.30 in case 5. TDP was used in four cases, but merely warfarin treatment was discontinued in one case. No complication developed, and all cases were discharged as INR returned to therapeutic levels. Patients absorbing warfarin may apply to the emergency with atypical hemorrhages, one of which is ocular type. Patients known to be treated with anticoagulants should be evaluated in detail as to clear or unclear hemorrhages.
\end{abstract}

J. Exp. Clin. Med., 2013; 30:389-393

\section{Keywords:}

Emergency medicine

International normalized ratio

Ocular hemorrhage

Warfarin

\section{Introduction}

As parallel to the increase in acute thromboembolic events leading to global mortality and morbidity, the number of patients treated with anticoagulants has also increased. However, the condition has resulted in an increase in hemorrhagic complications due to anticoagulant treatment. In a recent study, the rate of those on oral anticoagulant (OAC) treatment was reported to be about $1-1.7 \%$ of whole population and to become more increased day by day (Schurgers et al., 2004; Marzolini and Wynne, 2004). The major causes of such an increase, however, are population at older age and an elevation in the incidence of coronary arterial disease (CVDs). In light of clinical trials, global rate of hemorrhages in patients treated with OAC ranges between 2-13\% (Wiedermann and Stockner, 2008). A part of such patients may apply with fatal hemorrhages, while others may be encountered with minor multisystemic or atypical hemorrhages. In our study, five cases, especially admitting with orbital hemorrhages during the treatment of warfarin, were presented. Hemorrhagic complications of OAC treatment in patients were discussed with accompanying literature.

\section{Case-1}

A seventy six year old male patient applied to the emergency department with the complaints of swelling and hemorrhage on his right eye. In the history, it was found out that bruising was formed nearly ten days earlier under both eyes, started to spread around the eyes, and swelling was developed on right eye with palpebral bleeding a day earlier. The patient stated to be exposed to no traumas. It was figured out from the history that the patient had Atrial fibrillation (AF), had experienced cerebrovascular disease CVD two years ago, had undergone an operation of Perkutan Transluminal Koroner Anjioplasti (PTCA)-stent a year earlier and had been taking up kinapril, metoprolol, furacemid and warfarin. On admission, the general status and consciousness were stable, and the case was responsive to external stimuli. On physical examination, 


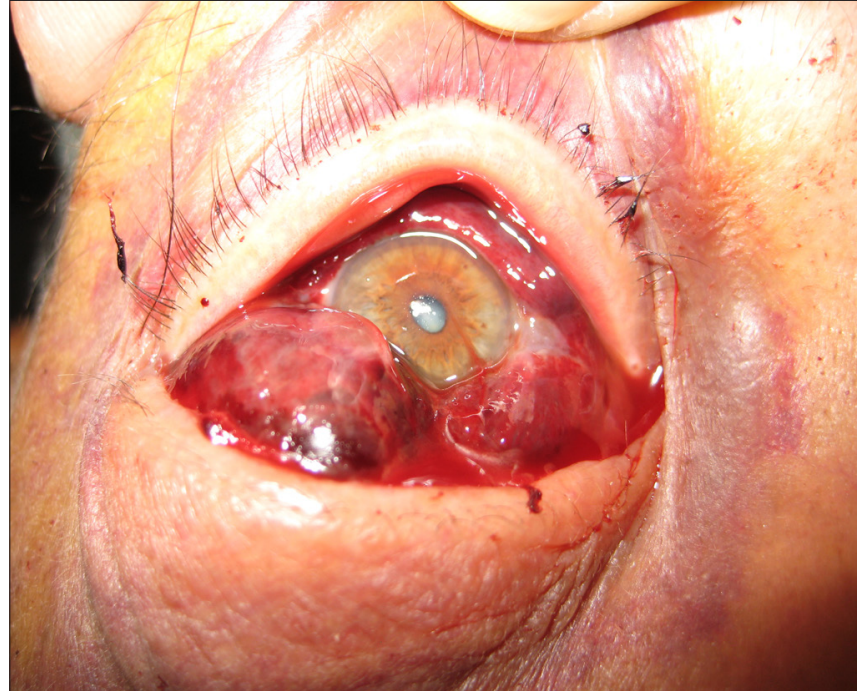

Fig. 1. Subconjonctival hematoma filling the lining of lower eyelid completely, and palpebral leaking of blood (Case-1).

blood pressure (BP), pulse (P), temperature $(\mathrm{T})$ and respiratory rate (RR) were $110 / 60 \mathrm{mmHg}$, pulse $(\mathrm{P}): 58 / \mathrm{min}, 37.0^{\circ} \mathrm{C}$ and respiratory rate $(\mathrm{RR})$ : $13 / \mathrm{min}$, respectively. Two-sided per orbital ecchymosed marked on right side, subconjonctival hematoma filling the lining of lower eyelid completely, and palpebral leaking of blood was detected on orbital examination (Fig. 1). No other pathological findings were encountered on other systemic examinations. In laboratory investigations, the findings were as follows: WBC:5.000 K/ $\mu \mathrm{L}, \mathrm{Hb}: 11.5 \mathrm{~g} / \mathrm{dL}$, Htc:35.2\%, Plt:193.000, INR:5.4, PT:49.9 sec and aPTT:51.9 sec. Biochemical parameters displayed no specialties. Atrial fibrillation was present on electrocardiography (ECG), and on orbital $\mathrm{CT}$, no pathological findings were detected on right lower eyelid, except for an increase of thickening. The treatment and follow-up were started for the patient in the emergency after stopping warfarin, and two-unit fresh frozen plasma (FFP) was administered. On examination at $7^{\text {th }}$ hour after FFP administration, INR, PT and aPTT were found as $3.26,33.2 \mathrm{sec}$ and $44.9 \mathrm{sec}$, respectively. No additional pathology was determined in the patient on consultation with the clinic of ophthalmology, and with the recommendations of rest and cardiologic and ophthalmologic follow-ups, the

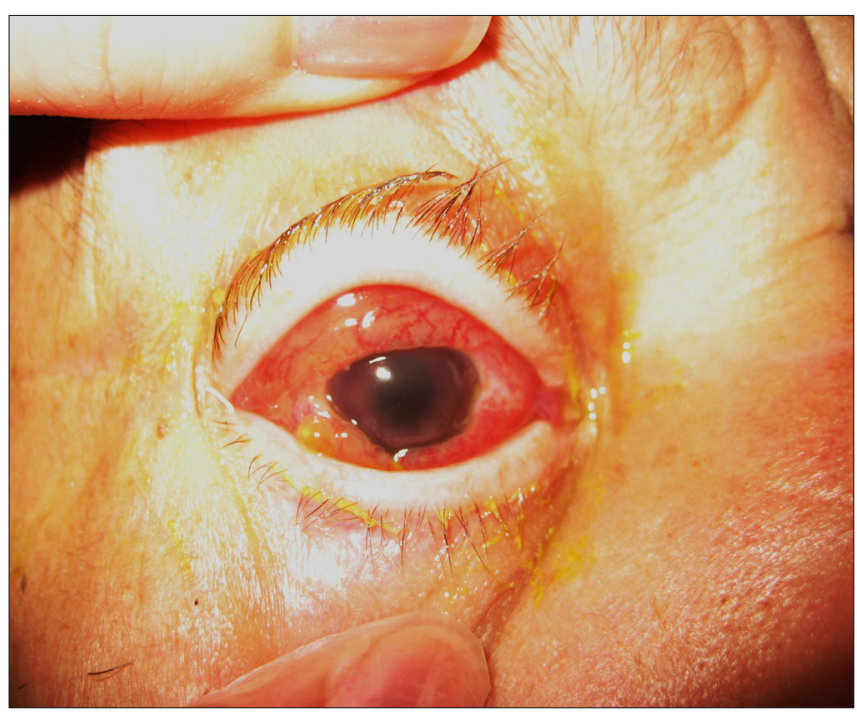

Fig. 2. Cornea on right eye was with edema, subconjonctival hyperemia and edema (Case2) patient was discharged on $2^{\text {nd }}$ day of hospitalization (INR: 2.28).

\section{Case-2}

A sixty five year old man applied to the emergency department with the complaints of pain and swelling on his right eye, and urinary bleeding. The patient reported hematuria and depth in urine over three days, and the severity of pain and swelling, and redness on right eye became more increased during the three day period. In the history, the patient were found out to have type 2 diabetes mellitus (DM), CVDs over 1.5 years and hypertension (HT), to be exposed to PTCA-stent, and on the use of Repaglinide, Rosiglitazone, Losartane, aspirin and warfarin. On his physical examination, general health status, consciousness and communicative skills were within normal limits, and BP, P, T and RR were 100/70 mmHg, 92/ $\min , 36.7^{\circ} \mathrm{C}$ and $16 / \mathrm{min}$, respectively. Cornea on right eye was with edema, subconjonctival hyperemia and edema were present, and light reflex was weak (Fig. 2), and a decrease in right eyesight was reported to occur during the last two days. Left eye were within normal limits, and on systemic examinations, skin was seen to be pale, and in heart, systolic murmur of 1-2/6 and dysrhythmia were detected. Respiration and abdomen were normal, solid melena was observed in rectal palpation, and ecchymosis in size of $10-12 \mathrm{~cm}$ was present in right gluteal area. However, the urine revealed gross hematuria. Laboratory findings were as follows: WBC:11.1 $\mathrm{K} / \mu \mathrm{L}, \mathrm{Hb}: 9.3 \mathrm{~g} / \mathrm{dL}$, Htc: 27.9\%, Plt:214.000, INR:8.3, aPTT:55.9 sec, blood glucose:230 mg/dL, Urea: $99 \mathrm{mg} / \mathrm{dL}$ and Cr:1.49 mg/dL, and no specifity was encountered in other biochemical parameters. ECG revealed no specifity, except AF. The patient was started to treat and follow up in intensive care unit (ICU) due to multiple systemic hemorrhages. The patient was administered $10 \mathrm{mg}$ of vitamine $\mathrm{K}$, two units of FFP and two units of erythrocyte suspension (ES), and in the orbital CT of patient with decreased INR of 2.40, common retinal detachment and subretinal hemorrhage was seen on right orbital posterior (Fig. 3). Consultation was performed with internal medicine and ophthalmology departments, and drug treatment was recommended. In follow-up there was no additional pathologies and patient discharged on thirth day of hospitalization.

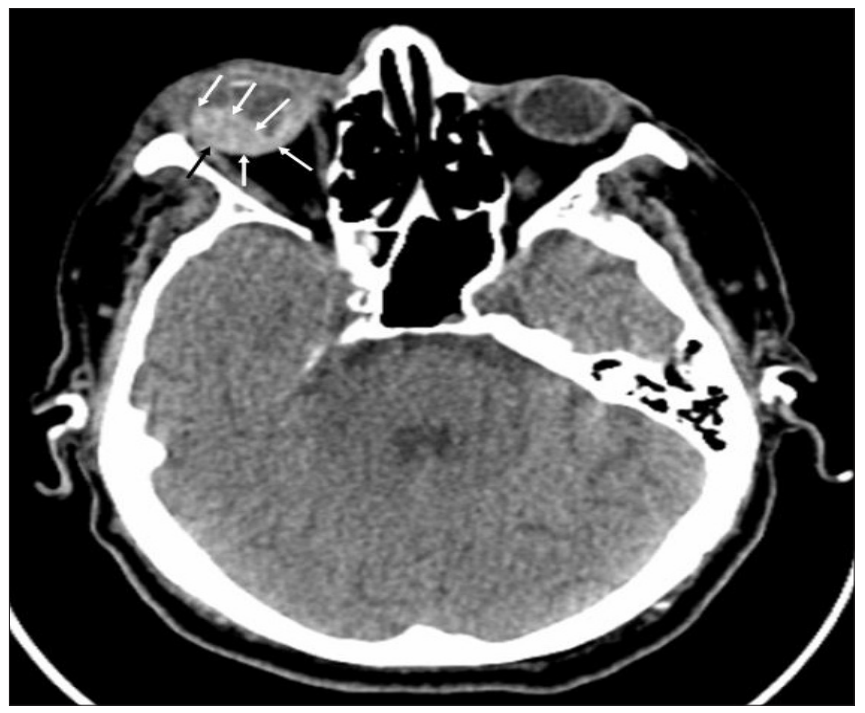

Fig. 3. Common retinal detachment and subretinal hemorrhage was seen on right orbital posterior (orbital CT) 


\section{Case-3}

A seventy year old woman applied to the emergency deparment with the complaints of redness, swelling, stinging and blood from mouth. Especially reporting blood from mouth while coughing up, the case also developed redness on her right eye. In the history, the patient with previous congestive heart failure (CHF), HT and AF declared she took in 1.25 $\mathrm{mg}$ of warfarin for five days per week over three months. On her physical examination, general health status, and consciousness were within normal limits, and BP, P, T and RR were $120 / 80 \mathrm{mmHg}, 75 / \mathrm{min}, 37.0^{\circ} \mathrm{C}$ and $13 / \mathrm{min}$, respectively. On head/neck examination, subconjonctival hemorrhage, edema and spectrum of scleral hematoma were present on right eye (Fig. 4). Left eye revealed no hemorrhagic findings. Movements of both eyes were free, IR was $+/+$, and no sight defects were detected. No gingival bleeding was observed in mouth; the heart was dysrhytmic and normocardial; while no specifity was observed on lung and abdominal examinations, petechial rashes were present on different part of the body, especially under both knees. On rectal examination, normal contamination of stool was seen. On laboratory readings, the findings were as follows: WBC: $10.000 \mathrm{~K} / \mu \mathrm{L}, \mathrm{Hb}: 11.9$ g/dL, Htc:37.2\%, Plt:177.000 K/mL, PT(INR) and aPTT: no coagulation, blood glucose: $123 \mathrm{mg} / \mathrm{dL}$, urea:59 mg/dL, Cr:1.3 mg/dL, ALT:534 U/L, AST:441 U/L and LDH:861 U/L. On abdominal ultrasonography (USG), stones in size of millimeter in gallbladder, and slight free fluid in perihepatic, perisplenic areas and lower quadrant. No marked pathology was detected in bulbus oculi and retrobulber areas on orbital CT. The case was hospitalized in ICU and followed up hemodynamically and hematologically with suspicion of increased liver enzymes and upper gastrointestinal tract (GIT) bleeding after consulting internal medicine, and with diagnosis of subconjonctival bleeding and scleral hematoma after consulting ophthalmology department. The patient was given five units of FFP, three units on day one and two units on day three. INR values were decreased to 3.55 and 3.46 , respectively. With improved general health status, no additional pathology and INR levels decreasing to normal, the patient was discharged with INR of 3.14 on day five after the hospitalization and recommended to comply with follow-ups.

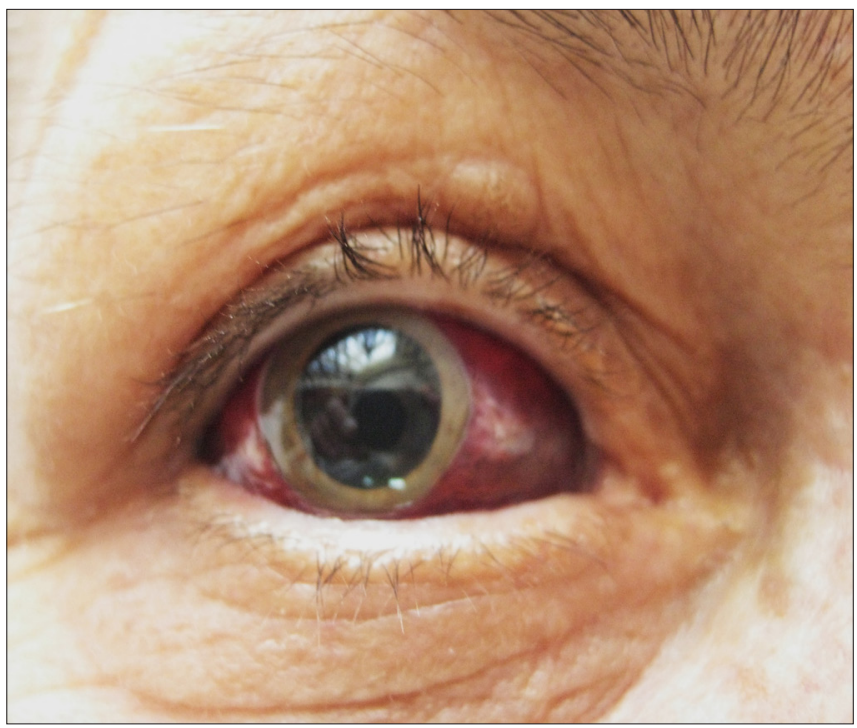

Fig. 4. Subconjonctival hemorrhage, edema and spectrum of scleral hematoma (Case-3).

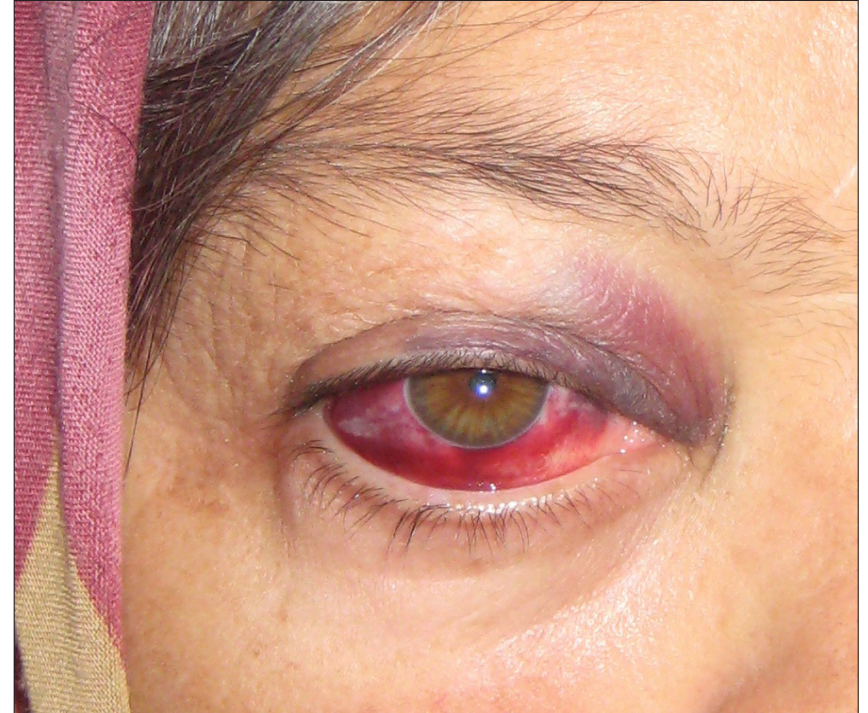

Fig.5. Subconjonctival and scleral hemorrhages, and edema (Case-4)

\section{Case-4}

A fifty five year old woman applied to the emergency with the complaints of redness and swelling on her red eye, bruising on right upper extremity and swelling on left knee. In the history of the patient reporting the complaints had started two days earlier, DM, HT and hyperlipidemia was present, and she was being treated with warfarin due to deep vein thrombosis (DVT). On her physical examination, general health status and consciousness were within normal limits, and BP, P, T and $\mathrm{RR}$ were $130 / 85 \mathrm{mmHg}, 86 / \mathrm{min}, 36.4^{\circ} \mathrm{C}$ and $14 / \mathrm{min}$, respectively. Subconjonctival and scleral hemorrhages, and edema were present on right eye (Fig. 5); ecchymosis in different sizes were found on right upper extremity, right shoulder and left gluteal regions; and, tenderness, swelling and hemarthrosis were detected on left knee. Movements of eyes were free, IR: $+/+$, and no defects related to eyesight were detected. No specifity were observed on heart, lung, abdominal and genital examinations, and laboratory findings were as follows: WBC:10.6 K/ $\mu \mathrm{L}, \mathrm{Hb}: 7.6 \mathrm{~g} / \mathrm{dL}, \mathrm{Htc}: 24.4 \%$, Plt:431.000 K/mL, PT(INR) and aPTT: no coagulation, blood glucose:158 mg/dL, urea:55 mg/dL, Cr:1.6 mg/dL, ALT:32 U/L and AST:20 U/L. No radiological test was performed. After consulting with department of orthopedics, the case was given three units of FFP and two units of ES, totally. INR values were, in turn, decreased to 3.05 and 2.30 during follow-ups. Fifty cc of fluid was drawn from left knee via arthrosynthesis by orthopedy department. In a inpatient hospital with emergency, the case was treated and followed, and no additional challenges were observed. The case whose INR values were decreased to therapeutic levels was discharged with recommendations to be followed-up in cardiovascular and orthopedy departments two days after the hospitalization.

\section{Case-5}

A eighty two year old man, while having no complaints a day earlier, applied to the emergency department with the complaints of redness on left eye as soon as waking up. In the history revealing KKY, HT, chronic bronchitis and AF, the patient was being treated with various drugs, mainly warfarin, ACE inhibitors, inhalers and beta agonists. Also, he had been exposed to surgeries due to bilateral cataract. 
On admission, general health status and consciousness were normal, and good communicative skills were observed. BP, $\mathrm{P}, \mathrm{T}$ and RR were $150 / 100 \mathrm{mmHg}, 78 / \mathrm{min}, 37^{\circ} \mathrm{C}$ and $16 /$ min, respectively. Hemorrhage was present on left eye (Fig. 6 ). The case reported no exposure to traumas and complaints with his eyesight. On systemic examination, heart was normocardic and dysrhytmic; respiratory sounds were hoarse; expirium was long; and abdomen was with no specifity. Skin examination revealed no petechial specifity, ecchimosis, etc. In stool and urine, no colour change was detected, and RT was within normal limits. Laboratory findings were seen as follows: WBC:5.6 K/ $\mu \mathrm{L}$, Hb:11.3 g/dL, Htc:24.4\%, Plt:289.000, PT(INR):5.3 aPTT: $38.2 \mathrm{sec,} \mathrm{blood} \mathrm{glucose:102}$ $\mathrm{mg} / \mathrm{dL}$, urea:28 mg/dL, Cr:1.1 mg/dL, ALT:27 U/L and AST:16 U/L. Without performing any radiological tests, the patient was consulted with ophthalmology department. Eye movements were free, IR:+/+, and no other pathology was determined except for subconjonctival hemorrhage. The case was recommended no other procedures except for topical drop and followed up for one day, discontinuing warfarin. The case with no other additional challenges was discharged with recommendation of ophtalmologic follow-up three days after the hospitalization.

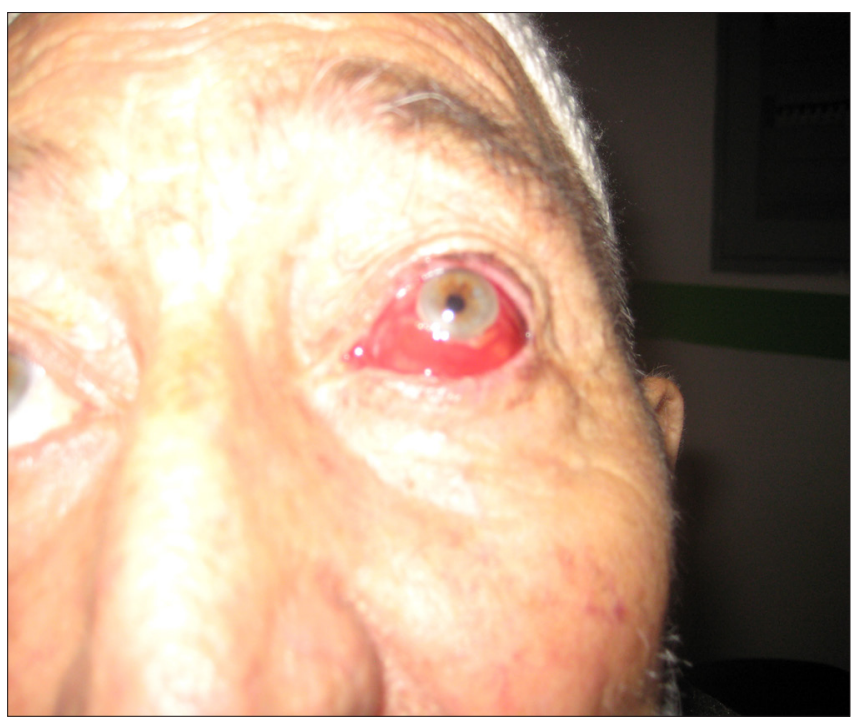

Fig. 6. Subconjonctival hemorrhage (Case5)

\section{Discussion}

The primary complication of OAC treatment is hemorrhages, and the risk of hemorrhage is related to the intensity of OAC treatment (Levine et al., 1995; Hirsh et al., 2003). Unfortunately, to maintain the equilibrium between the dose he prevent patients on the use of OAC from thromboembolic complications and the dose to inhibit the occurrence of hemorrhages still remains a serious challenge. Major risk of hemorrhages during $\mathrm{OAC}$ treatment as reported to be around $1-7.4 \%$ in cohort studies (Wiedermann and Stockner, 2008). More than $1 \%$ of hemorrhages due to OAC treatment may result in deaths owing to major bleedings. Many factors leading to hemorrhages have been suggested, and of these, the most significant is the intensity of OAC treatment. In several studies, it was suggested that especially patients with INR $>5$ are at a larger risk due to hemorrhagic complications (Ansell et al., 2001). As consistent with the findings of aforementioned literature, of five patients in our study, two were with immeasurable INR scores, and three were with INR 5. Factors related to patient, notably old age, are suggested to cause serious predisposition to hemorrhages, and patients at the age of 65 and over were reported to be more vulnerable to major bleedings (Landefeld et al., 1989). As opposed to such evidence, in a study performed with a large population (except for those at the age of 80 and over), a marked factor was reported not to be present for risk of bleeding for patients treated with warfarin (Fihn et al., 1996). Therefore, the caudation still remains unclear. Among the participants in our study, one was at the age of 55, and others were 65 and over. In context of hemorrhages, old age may be suggestive of indirect effects like the incompliance with treatment due to accompanying diseases, drug absorption at a higher rate and a decrease in cognitive functions. In addition, accompanying disorders and drugs absorbed are among other factors increasing propensity to hemorrhages (Levine et al., 1995). Such accompanying factors as history of GIT bleedings, hypertension, experienced CVE, AF, anemia and renal failure, and synergic effect of warfarin with other drugs, mainly aspirin and other Nonsteroidal anti-inflammatory drugs (NSAIDs) are stated to increase the tendency to hemorrhages (Ansell et al., 2001; Ahmed et al., 2008). All the patient in our study had accompanying chronic disorders, and were taking up several drugs, mainly aspirin. In literature, various ocular hemorrhages (subretinal, conjonctival, vitreal and choroideal) related to use of warfarin were reported (Marie and Bodack, 2007). In a study performed in ophthalmology department with patients treated with OAC, $3 \%$ of patients were determined to be with intraretinal hemorrhages, and emphasized that risk of hemorrhage is decreased without susceptible factors (Superstein et al., 2000). In one of our participants, history of cataract surgery was detected, and other participants were no history of eye disorders.

On condition that overdoses of warfarin (INR over therapeutic limits) is used, the management of patients may change under such conditions as level of INR in patients, and whether hemorrhage ,if any, is minor or major, or life threatening (Hirsh et al., 2008). On condition that serious or life threatening hemorrhage is present, discontinuation of warfarin and administration of parenteral vitamin K, FFP or prothrombin complex concentration, or recombinant FVIIa are proposed. However, according to INR levels, skipping doses or administration of oral vitamin $\mathrm{K}$ are suggested for those with no hemorrhages, but as to level of hemorrhages, a discrepancy still exists, and no consensus is present among healthcare professionals. While various researchers classify as major, minor or fatal, others have grouped hemorrhages into solely major and minor (Ansell et al., 2001). Although researchers are present, accepting fatal, intracranial or retroperitoneal hemorrhages and those leading to a sudden decrease in $\mathrm{Hb}$ or requiring transfusion and hospitalization as major bleedings, no common criteria or consensus exist in the differential diagnosis of hemorrhages. In our cases, except one, TDP was chosen due to localization of hemorrhages, need for transfusion, accompanying other systemic bleedings and high level of INR. No challenges were observed in our cases, stemming from use of TDP during follow-ups. 


\section{Conclusion}

Patients under the treatment of warfarin may admit to clinics with fatal or non-fatal hemorrhagic complications, as well as rare or atypical hemorrhages, due to many different predisposing factors. Among such factors are ocular hemorrhages. In those admitting to in emergency departments with the complaint of red eyes, whether with the history of traumas or not, overdose or overuse of anticoagulants should be taken into consideration, and patients should definitely be evaluated in terms of visual functions. In problematic cases, additional pathologies should be ruled out via orbital CT. Further, those known to be treated with OAC should be investigated in detail, whatever the complaint is, as to clear or unclear bleedings.

\section{REFERENCES}

Ahmed, A., Stephens, J.C., Kaus, C.A., Fay, W.P., 2008. Impact of preemptive warfarin dose reduction on anticoagulation after initiation of trimethoprim-sulfamethoxazole or levofloxacin. J. Thromb. Thrombolysis. 26, 44-48.

Ansell, J., Hirsh, J., Dalen, J., Bussey, H., Anderson, D., Poller, L., Jacobson, A., Deykin, D., Matchar, D., 2001 . Managing oral anticoagulant therapy. Chest. 119, 22-38.

Fihn, S.D., Callahan, C.M., Martin, D.C., McDonell, M.B., Henikoff, J.G., White, R.H.,1996.The risk for and severity of bleeding complications in elderly patients treated with warfarin. The National Consortium of Anticoagulation Clinics. Ann Intern Med. 124, 970-979.

Hirsh, J., Fuster, V., Ansell, J., Halperin, J.L., 2003. American Heart Association/American College of Cardiology Foundation Guide to Warfarin Therapy. Circulation. 107, 1692-1711.

Hirsh, J., Guyatt, G., Albers, G.W., Harrington, R., Schünemann, H.J., 2008. Antithrombotic and thrombolytic therapy: ACCP guidelines; Executive summary: American College of Chest Physicians Evidence-Based Clinical Practice Guidelines (8th Edition). Chest. 133, 71-109.

Landefeld, C.S., Goldman, L., 1989. Major bleeding in outpatients treated with warfarin: incidence and prediction by factors known at the start of outpatient therapy. Am. J. Med. 87, 144-152.

Levine, M.N., Raskob, G., Landefeld, S., Hırs J., 1995. Hemorrhagic complications of anticoagulant treatment. Chest. 108, $276-290$.

Marie, I., Bodack, O.D., 2007. A warfarin-induced subconjunctival hemorrhage. Optometry. 78, 113-118.

Marzolini, M., Wynne, H., 2002.Should patients manage their own oral anticoagulation therapy? Rev. Clin. Gerontol. 12, $275-281$.

Schurgers, L.J., Aebert, H., Vermeer, C., Bültmann, B., Janzen, J., 2004. Oral anticoagulant treatment: Friend or foe in cardiovascular disease? Blood. 104, 3231-3232.

Superstein, R., Gomolin, J.E., Hammouda, W., Rosenberg, A., Overbury, O., Arsenault, C., 2000. Prevalence of ocular hemorrhage in patients receiving warfarin therapy. Can. J. Ophthalmol. 35, 385-389.

Wiedermann, C.J., Stockner, I., 2008. Warfarin-induced bleeding complications-clinical presentation and therapeutic options. Thromb. Res. 2, 13-18. 